\title{
Serum nesfatin-1 and galanin concentrations in the adult with metabolic syndrome
}

\author{
Relationships to insulin resistance and obesity
}

Maryam N. Alotibi, MMS, Amina M. Alnoury, MD, Amani M. Alhozali, MD, SSC-Med.

\begin{abstract}
الأهداف : للتحقق من مستويات مصل النسفاتين1- والجلانين

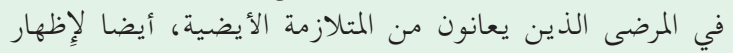

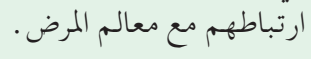

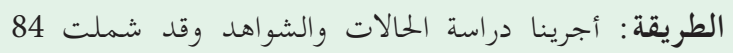

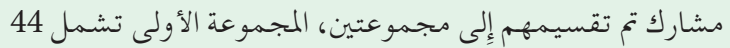

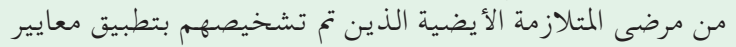

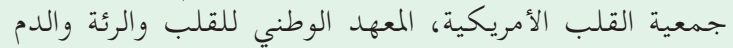

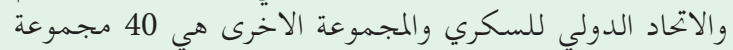

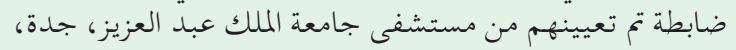

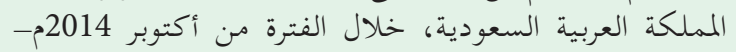

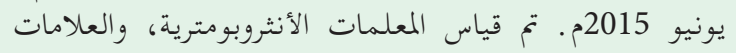
البيو كيميائية وكذلك النيسفاتن 1 - والجلانين.

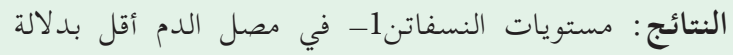

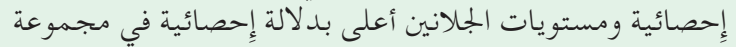

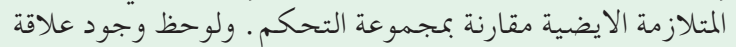

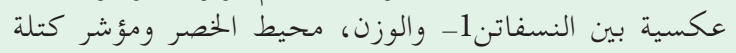

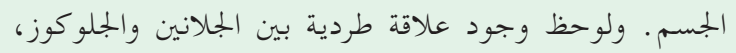

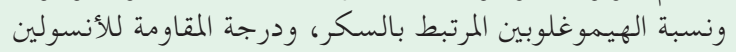

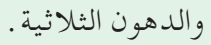

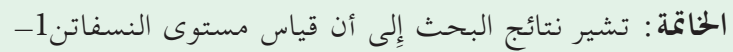

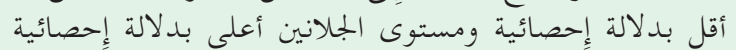

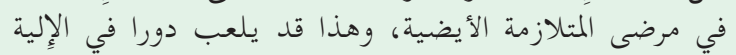

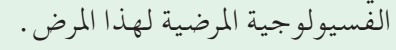

Objectives: To investigate the serum levels of nesfatin-1 and galanin in patients with metabolic syndrome (MetS), and also to show their association with the parameters of the disease.

Methods: We performed a case-control study with 84 participants (44 patients with MetS diagnosed according to the American Heart Association/ National Heart, Lung, and Blood Institute and International Diabetes Federation criteria and 40 control group) were recruited from King Abdulaziz University Hospital, Jeddah, Saudi Arabia, between October 2014 and June 2015. Anthropometric parameters, biochemical markers as well as nesfatin-1 and galanin were measured.

Results: Nesfatin-1 levels were found to be significantly lower and galanin levels significantly higher in MetS group compared to the control group. A significant negative correlation between serum nesfatin-1 and weight, waist circumference, and body mass index were observed. A significant positive correlation between serum galanin and with fasting blood glucose, glycosylated hemoglobin, homeostasis model assessment-insulin resistance, and triglycerides.

Conclusion: Our findings indicated a lower level of nesfatin-1 and a higher level of galanin in patients with MetS, suggesting a role of these neuropeptides in the pathogenesis of this disease.

Saudi Med J 2019; Vol. 40 (1): 19-25

doi: 10.15537/smj.2019.1.22825

From the Department of Medicine, King Abdulaziz University Hospital, Jeddah, Kingdom of Saudi Arabia.

Received 19th June 2018. Accepted 14th November 2018.

Address correspondence and reprint request to: Ms. Maryam N. Alotibi, Clinical Biochemistry Department, King Abdulaziz University, Jeddah, Kingdom of Saudi Arabia. E-mail: mary.n_08@windowslive.com ORCID ID: orcid.org/0000-0003-4533-2589

Disclosure. This study was funded by King Abdulaziz City for Science and Technology (KACST), Riyadh, Saudi Arabia (Research No. 73-36). 
M etabolic syndrome (MetS) denotes a group of metabolic risk factors for cardiovascular diseases and diabetes, including central obesity, elevated blood glucose levels and/or hyperinsulinemia, dyslipidemia, and elevated blood pressure. ${ }^{1}$ Its prevalence is rapidly increasing, and it has become an epidemic health problem. ${ }^{2}$ The prevalence of MetS among Saudi adults ranges from $16 \%$ to $40 \%$ according to the study area and definition utilized. ${ }^{3}$ The exact role of these physiopathological causes underlying MetS has not been fully elucidated. Many peptide hormones and neural satiety signals that were first detected in the hypothalamus have been shown in many peripheral tissues, including adipose tissue and the gastrointestinal tract. These peptides play essential roles in body weight regulation, feeding conduct, and body energy balance. ${ }^{4-6}$

One of these neuropeptides is nesfatin-1, which was identified in 2006 as an anorexigenic neuropeptide; nesfatin-1 is an 82 amino acids peptide derived from the precursor hypothalamic protein; nucleobindin-2 (NUCB2) by posttranslational modification. ${ }^{7}$ It is widely expressed in the central nervous system and several tissues, such as the pancreatic islet cells, pituitary gland, adipose tissue, and stomach. ${ }^{8}$ It has a key role in regulating food intake, body weight, appetite stimulation and body energy balance. ${ }^{9,10}$ The relationship between nesfatn-1 and MetS is still studied. It was found to have anti-hyperglycemic and insulinotropic properties in addition to its anorexigenic effects. ${ }^{11}$ Nesfatin-1 enhances glucose-stimulated insulin secretion from $\beta$-cells through the direct interaction involving $\mathrm{Ca} 2+$ entry via (L-type calcium channels). ${ }^{12}$ Increasing evidence support the participation of this neuropeptide in the regulation of metabolism. Investigation of the association of circulating NUCB2/nesfatin-1 with body weight showed that circulating plasma NUCB2/ nesfatin-1 levels were lower in cases of anorexia nervosa and higher in obese persons. ${ }^{13,14}$

Another neuropeptide is galanin, a 29-amino acid peptide that was discovered in 1983 in the porcine intestine as an orexigenic neuropeptide; it is mainly synthesized in the central and peripheral nervous systems and gastrointestinal tract. ${ }^{15,16}$ It exerts its effects through the activation of galanin receptors 1, 2, and 3. It plays an important role in the regulation of energy balance and modulation of food intake. ${ }^{17}$ Existing evidence propose that galanin could contribute to the event of MetS in adults. Several animal and clinical studies reported that galanin improves insulin sensitivity and enhance glucose clearance in adipose tissue, skeletal muscle and heart muscle by glucose transporter type 4 receptors. It also suppresses glucose-stimulated insulin secretion in both human and animal models. ${ }^{18-20}$ Galanin concentration was noted to be significantly increased in type 2 diabetes mellitus (T2DM), gestational diabetes mellitus (GDM) and obese subjects. ${ }^{18,21}$

Due to the lack of a consensus on nesfatin-1 and galanin concentrations and their relationship to anthropometric and metabolic parameters in case of MetS, we aim to investigate the levels of galanin and nesfatin-1 in patients with MetS and explore their association with the parameters of the disease.

Methods. This was a case-control study that was conducted between October 2014 and June 2015 in the Internal Medicine Outpatient Clinic at King Abdulaziz University Hospital, Jeddah, Saudi Arabia. It included 84 participants who were further divided into 2 groups; group 1 included $44 \mathrm{MetS}$ patients (23 females and 21 males), while group 2 was comprised of non-MetS control group hospital based (22 females and 18 males).

We selected control participants from dentist, orthopedic, and ophthalmology clinic through purposive sampling technique. The participants' ages ranged from 35 to 75 years old. Each subject was medically examined and interviewed using the standardized questionnaire in relation to the history of disease and medications. Subjects with renal disease, thyroid disorder, pregnancy or liver disease were excluded. Informed consent was obtained from the subjects. The study protocol was approved by the Biomedical Ethics Research Committee of the Faculty of Medicine, King Abdulaziz University, Jeddah, Saudi Arabia.

All participants were screened for MetS based on the criteria set by the American Heart Association/National Heart, Lung, and Blood Institute (AHA/NHLBI) and International Diabetes Federation (IDF). ${ }^{22}$ At least 3 of the following 5 metabolic abnormalities had to be present: 1) central obesity, as indicated by waist circumference (WC) (males: $\geq 80 \mathrm{~cm}$ and females: $\geq 94$ $\mathrm{cm})$; 2) impaired fasting blood glucose (FBG) $\geq 5.5$ $\mathrm{mmol} / \mathrm{L}$ or current drug treatment for elevated glucose; 3) raised triglycerides (TG) $\geq 1.7 \mathrm{mmol} / \mathrm{L}$ or treatment for this lipid abnormality; 4) reduced glycosylated hemoglobin (HbA1c) level (males: $<1.03 \mathrm{mmol} / \mathrm{L}$ and for females: $<1.3 \mathrm{mmol} / \mathrm{L}$ ) or treatment for this lipid abnormality; and 5) hypertension (systolic and/or diastolic blood pressures $\geq 130 / 85 \mathrm{~mm} \mathrm{Hg}$ or who were receiving antihypertensive treatment).

Waist circumference, body weight, and height were assessed for all the participants. Body mass index (BMI) was calculated as weight in kilograms divided by height (in meters) squared. Waist circumference was recorded 
in centimeters. Blood pressure was measured by the standard procedure.

Venous blood samples (total of $5 \mathrm{~mL}$ blood) were drawn from the participants in the morning after ( $\approx 120-14$ hours) fasting. The serum samples of each participant were collected into a vacutainer serum separator tubes and separated into clean dried Eppendorf tubes and stored at ${ }^{-} 80^{\circ} \mathrm{C}$ until needed.

All biochemistry measurements were assayed at KAUH's clinical laboratory. Total cholesterol (TC), high-density lipoprotein cholesterol (HDL-C), low-density lipoprotein cholesterol (LDL-C), TG, FBG, insulin, and HbA1c were assessed using the Dimension Vista System (Dimension Vista1500, Healthcare Company, New York, USA). Fasting blood glucose was performed by enzymatic hexokinase method, while TC, HDL-C, LDL-C, and TG were assessed by the enzymatic methods. Glycosylated hemoglobin (HbAlc) was determined by a turbidimetry immunoassay. Serum insulin was determined using a direct chemiluminescent technology immunoassay (ADVIA Centaur Insulin Assay). Insulin resistance was estimated using homeostasis model assessment-insulin resistance (HOMA-IR) with the following formula: fasting plasma glucose $(\mathrm{mmol} / \mathrm{L}) \times$ fasting serum insulin $(\mu \mathrm{U} / \mathrm{mL}) / 22.5$.

The concentration of nesfatin-1 in serum was determined using a Human Nesfatin-1 ELISA kit
(Cusabio Biotech, Wuhan, China). This test kit is effective in the range of $31.25-2,000 \mathrm{pg} / \mathrm{ml}$. While the concentration of galanin in serum was determined using a Human Galanin ELISA kit (Cusabio Biotech, Wuhan, China). This test kit is effective in the range of $4.7-300 \mathrm{pg} / \mathrm{ml}$.

Statistical analysis. Data were entered and analyzed using the Statistical Package for Social Sciences (Version 20, SPSS Inc., Chicago, IL, USA). Descriptive data were given as the mean \pm standard deviation (SD). Levenes test was applied to see the equality of variances. Comparison of the means between 2 groups was made with the independent-samples t-test, whereas the differences in percentages of categorical variables were assessed using a Chi-square test. The relationship between serum nesfatin-1, galanin concentrations, and components of MetS were calculated using pearson's correlation test. For all the tests, the differences were considered significant at a $p<0.05$.

Results. A total of 84 subjects were enrolled in this case-control study. The mean age of cases and controls was $54.05 \pm 9.28$ and $57.05 \pm 8.53$ years. The subjects were stratified into 2 groups: MetS group $(n=44)$ (male: 21 and female: 23$)$ and the control group $(n=40)$ (male: 18 and female: 22). The clinical and biochemical characteristics of the groups are summarized in Table 1. Significant differences $(p<0.05)$ between participants

Table 1 - The clinical and biochemical characteristics of the studied groups.

\begin{tabular}{lccc}
\hline Variables & $\begin{array}{c}\text { Control subjects } \\
(\mathbf{n}=40)\end{array}$ & $\begin{array}{c}\text { Metabolic syndrome } \\
\text { patients }(\mathbf{n}=44)\end{array}$ & $P$-value \\
\hline Age $($ year $)$ & $54.05 \pm 9.28$ & $57.05 \pm 8.53$ & 0.127 \\
Height $(\mathrm{cm})$ & $159 \pm 0.08$ & $162 \pm 0.08$ & 0.070 \\
Weight $(\mathrm{kg})$ & $73.97 \pm 13.62$ & $83.02 \pm 15.22$ & 0.005 \\
BMI $\left(\mathrm{kg} / \mathrm{m}^{2}\right)$ & $29.30 \pm 5.97$ & $31.29 \pm 4.91$ & 0.097 \\
Waist circumference $(\mathrm{cm})$ & $90.60 \pm 11.68$ & $108.05 \pm 11.15$ & 0.000 \\
Systolic blood pressure $(\mathrm{mm} \mathrm{Hg})$ & $121.35 \pm 11.96$ & $141.18 \pm 17.83$ & 0.000 \\
Diastolic blood pressure $(\mathrm{mm} \mathrm{Hg})$ & $73.63 \pm 8.98$ & $74.86 \pm 11.41$ & 0.585 \\
Fasting blood glucose (mmol/l) & $5.28 \pm 0.62$ & $8.81 \pm 2.72$ & 0.000 \\
HbA1C $(\%)$ & $5.62 \pm 0.39$ & $7.69 \pm 1.57$ & 0.000 \\
Insulin (mlU/L) & $12.14 \pm 5.88$ & $15.81 \pm 7.73$ & 0.017 \\
HOMA-IR & $2.87 \pm 1.46$ & $6.41 \pm 4.35$ & 0.000 \\
Total cholesterol (mmol/L) & $5.22 \pm 0.96$ & $4.49 \pm 1.04$ & 0.000 \\
Triglycerides (mmol/L) & $1.29 \pm 0.54$ & $1.62 \pm 0.73$ & 0.024 \\
HDL-C (mmol/L) & $1.41 \pm 0.36$ & $1.31 \pm 0.54$ & 0.366 \\
LDL-C (mmol/L) & $3.24 \pm 0.75$ & $2.84 \pm 0.93$ & 0.034 \\
Nesfatin-1 (pg/ml) & $2017.14 \pm 871.34$ & $1659.91 \pm 1078.35$ & 0.035 \\
Galanin (pg/ml) & $35.99 \pm 30.57$ & $53.12 \pm 35.79$ & 0.015 \\
\hline \multicolumn{2}{l}{ Values are represented as mean \pm standard deviation. $p<0.05$ is significant, BMI- body mass index, HbA1C } \\
- glycosylated hemoglobin, HOMA-IR - homeostasis model assessment for insulin resistance, HDL-C - high \\
$\quad$ density lipoprotein-cholesterol, LDL-C - low density lipoprotein-cholesterol \\
\hline
\end{tabular}


Table 2 - Frequencies and percentages of metabolic syndrome risk factors among the studied population.

\begin{tabular}{lrrrr}
\hline MetS risk factors & \multicolumn{2}{c}{$\begin{array}{c}\text { Controls } \\
(\mathbf{n}=\mathbf{4 0})\end{array}$} & $\begin{array}{c}\text { MetS } \\
(\mathbf{n}=44)\end{array}$ & $P$-value \\
\hline Central obesity & 19 & $(47.5)$ & $42(95.5)$ & 0.000 \\
High FBG & 4 & $(10.0)$ & $44(100.0)$ & 0.000 \\
Hypertension & 1 & $(2.5)$ & $41(93.2)$ & 0.000 \\
Low HDL-C & 2 & $(5.0)$ & $19(43.2)$ & 0.000 \\
High triglyceride & 4 & $(10.0)$ & $18(40.9)$ & 0.001 \\
\hline \multicolumn{4}{l}{$\begin{array}{l}\text { Values are represented as number and (percentage). HDL-C - high } \\
\text { density lipoprotein-cholesterol, FBG - fasting blood glucose }\end{array}$} \\
\hline
\end{tabular}

with MetS and controls were found for the components of MetS (WC, systolic blood pressure [SBP], TG, and FBG). There was no significant difference $(p>0.05)$ between groups in age, height, BMI, diastolic blood pressure (DBP), or HDL-C. Regarding the analysis of serum nesfatin-1 and galanin levels. The data showed significantly lower levels of nesfatin-1 in MetS patients than in control subjects, $p<0.05$. In contrast, the mean serum levels of galanin were significantly higher in patients with MetS compared with the control subjects.

The prevalence of MetS risk factors among the controls and MetS patients were studied using the AHA/NHLBI and IDF criteria, as shown in Table 2. The prevalence of all risk factors (high FBG, hypertension, low HDL-C levels, abdominal obesity, and high TG) was significantly higher in the MetS group than in the control group $(p<0.001)$. Among the individual components of MetS, the most prevalent risk factors in patients were the high FBG levels, abdominal obesity, and hypertension while the least prevalent were low serum HDL-C and high serum TG. In the control group, the most prevalent risk factor was abdominal obesity, indicating increased obesity among the studied population.

Table 3 reveals that galanin correlated positively with FBG, HbA1C, HOMA-IR, and TG $(p<0.05)$. Nesfatin-1 correlated negatively with weight, WC, and BMI $(p<0.05)$. No correlation between galanin and nesfatin-1 was observed.

Discussion. Metabolic syndrome is one of the most complex and heterogeneous disorders. It is characterized by abdominal obesity, hypertension, hyperglycemia, and lipid metabolic abnormalities. It has been identified as an independent cardiovascular diseases risk beyond the sum of its individual components. ${ }^{1}$ The prevalence of MetS is increasing in most countries; one possible reason for this is the epidemic of obesity in most populations. ${ }^{2}$ Neuropeptides probably play a critical role in MetS. In
Table 3 - Correlation between serum galanin, nesfatin-1 levels and variables in the studied population.

\begin{tabular}{lcccc}
\hline Variables & \multicolumn{2}{c}{ Galanin } & \multicolumn{2}{c}{ Nesfatin-1 } \\
& $r$ & $P$-value & $r$ & $P$-value \\
\hline Clinical parameters & & & & \\
Age & 0.144 & 0.190 & 0.200 & 0.069 \\
Height & 0.097 & 0.381 & -0.102 & 0.357 \\
Weight & 0.004 & 0.974 & -0.348 & 0.001 \\
BMI & -0.063 & 0.566 & -0.282 & 0.009 \\
WC & 0.072 & 0.518 & -0.376 & 0.000 \\
SBP & -0.024 & 0.829 & -0.072 & 0.515 \\
DBP & 0.032 & 0.770 & -0.071 & 0.520 \\
Biochemical parameters & & & & \\
FBG & 0.322 & 0.003 & -0.172 & 0.118 \\
HbA1C (\%) & 0.289 & 0.008 & -0.121 & 0.273 \\
Insulin & 0.168 & 0.127 & -0.095 & 0.288 \\
HOMA-IR & 0.255 & 0.019 & -0.136 & 0.217 \\
Total cholesterol & 0.003 & 0.976 & -0.110 & 0.318 \\
Triglycerides & 0.243 & 0.026 & -0.191 & 0.081 \\
HDL-C & -0.166 & 0.131 & -0.057 & 0.603 \\
LDL-C & 0.045 & 0.681 & -0.164 & 0.136 \\
Nesfatin-1 (pg/ml) & 0.085 & 0.440 & 0.085 & 0.440 \\
\hline
\end{tabular}

$r$ - Pearson's correlation, $p<0.05$ is significant, WC - waist circumference, BMI - body mass index, SBP - systolic blood pressure, DBP - systolic blood pressure, FBG - fasting blood glucose, HbA1c - glycosylated hemoglobin,

HOMA-IR - homeostasis model assessment for insulin resistance, HDL-C - high density lipoprotein-cholesterol, LDL-C - low density lipoprotein-cholesterol

recent years, the number and varieties of neuropeptides have been growing; therefore, understanding the diverse effects of these identified neuropeptides is important. ${ }^{4,5}$ In this study, our primary aim was to investigate the levels of nesfatin-1 and galanin in patients with MetS, as defined by the AHA/NHLBI and IDF criteria and compare their serum levels with those of controls. We also assessed the relationship between these 2 neuropeptides and biochemical markers of MetS.

In the present study, TG, FBG, SBP, and WC (risk factors for MetS) were significantly higher in patients with MetS when compared with the control group. Neither DBP nor HDL-C was significantly different between the 2 groups, and this may have been due to drug treatment for hypertension and dyslipidemia.

Our observations showed that serum galanin concentrations were higher in MetS patients than in their control subjects. No previous study has analyzed galanin among adults with MetS, but has only been done in patients with individual components of MetS. In this study, a significant positive correlation was determined between serum galanin and FBG, HbA1C, and HOMA-IR (parameters indicating diabetes). This 
is in accordance with previous reports that showed a statistically significant positive correlation between galanin concentrations and FBG content in healthy individuals and patients with T2DM during the glucose tolerance test, pregnant women with GDM and non-pregnant women with T2DM. ${ }^{21}$ Moreover, a positive association was shown between the plasma concentrations of galanin and $\mathrm{HbAlc}$ in children with T1DM. ${ }^{23}$ Zhang et $\mathrm{al}^{24}$ showed a significant positive correlation between galanin and HO MA-I R. These data seem to indicate that the enhanced galanin concentration may be a consequence of increased serum glucose. Regarding the lipid profile, Fang et $\mathrm{al}^{25}$ reported that a statistically significant positive correlation between galanin concentrations and TG in obese participants. Similarly, in the present study serum galanin levels were significantly positive correlated with TG.

Our data did not show a correlation between the serum galanin level and body weight, BMI, or WC. In the literature, several studies closely related galanin to body weight and obesity via regulation of food intake in animals and humans. ${ }^{26}$ Plasma galanin levels were reported to be increased in obese females versus control subjects, especially in individuals with a BMI $>31$. In comparison, the concentrations of galanin were reduced in lean women compared with healthy subjects, suggesting that circulating concentrations of galanin are increased in individuals with a greater visceral fat mass. ${ }^{27}$ These results are not in agreement with the findings of this study, which may be explained according to the small size of the studied population or high percentage of obese persons in the patient $(95.5 \%)$ and control groups $(47.5 \%)$.

The relationship between galanin and hypertension has not been completely understood. Some research studies have reported the implications of galanin in the regulation of blood pressure and heart rate in animals. ${ }^{28}$ A recent study found that the plasma galanin concentrations were reduced in obese individuals with hypertension compared with age- and BMI-matched obese control groups. Furthermore, the plasma galanin concentration was negatively correlated with DBP in obese subjects with hypertension. ${ }^{25,29}$ However, our study found no correlation between the serum galanin level and DBP and a non-significant negative correlation with SBP. Regarding the other neuropeptide, nesfatin-1, we observed significantly lower nesfatin-1 concentrations in individuals with MetS versus a control. In previous study, there has been only one study that also showed lower levels of nesfatin-1 in MetS patients. ${ }^{30}$ In the present study, no correlation between the nesfatin-1 level and FBG, HbA1C, fasting insulin, and HOMA-IR were observed. The important role of nesfatin-1 in the metabolism of glucose and insulin has been reported in several studies. However, the results of these studies on the relationships between nesfatin-1 and diabetes or insulin resistance have revealed inconsistent findings. Su et a ${ }^{31}$ showed a decrease in plasma glucose levels after nesfatin- 1 injection in rats, and they suggested that the anti-hyperglycemic effect of nesfatin-1 probably occurs through the insulin signal pathways, but the exact mechanism was not elucidated. Some studies have shown reduced nesfatin- 1 concentrations in patients with T2DM and high nesfatin-1 levels in patients with T1DM compared with healthy adults. ${ }^{32}$ Furthermore, nesfatin-1 concentrations were also reported to be decreased in patients with GDM compared with control pregnant women. ${ }^{33}$ Deniz et a ${ }^{34}$ determined that serum nesfatin-1 concentrations were lower in women with polycystic ovary syndrome who had insulin resistance compared with the control group. However, other studies have observed a high nesfatin-1 concentration in patients with T2DM and patients with impaired glucose tolerance, with a positive correlation between plasma nesfatin-1 concentrations and FBG, plasma insulin, second-hour blood glucose after a glucose load, and HOMA-IR. ${ }^{35}$ Moreover, intravenous infusion of glucose significantly increased basal nesfatin-1 concentrations in healthy adult subjects. ${ }^{36}$

Our study demonstrated negative correlations between nesfatin-1 and WC, BMI, and body weight (obesity parameters). An early study has shown an inverse correlation between nesfatin-1 concentrations and BMI in non-obese subjects. ${ }^{37}$ Moreover, another study observed a significant decrease in the levels of the fasting nesfatin-1 in the subjects with higher BMI compared to control group and showed a negative correlation between nesfatin-1 and BMI, body fat percentage and body fat weight. ${ }^{38}$ Furthermore, in patients with a non-alcoholic fatty liver disease, serum nesfatin-1 concentrations in obese subjects were significantly reduced compared with non-obese subjects. ${ }^{39}$ Another study has proved the positive correlation between BMI and nesfatin- 1 levels in obese and overweight subjects. ${ }^{10}$

Regarding the lipid profile, there has been no prior report in the literature concerning the relationship between the lipid profile and nesfatin-1 concentrations in MetS patients. One study found that nesfatin-1 was negatively correlated with TG in patients with hypothyroidism. ${ }^{40}$ Another found a positive correlation between nesfatin-1 concentrations and HDL-C in patients with T2DM. ${ }^{41}$ In the current study, we 
did not observe any correlation between nesfatin-1 concentrations and HDL-C, LDL-C, TC, or TG. Concerning the effect of nesfatin-1 on blood pressure, the results of the different studies are contradictory. Intravenous injection of nesfatin-1 in rats and mice resulted in a significant elevation of blood pressure. ${ }^{42}$ Moreover, Zhao et $\mathrm{al}^{43}$ found a significant increase in plasma nesfatin-1 levels in patients with hypertension versus control subjects, as well as positive correlations with SBP and DBP, especially in overweight/obese hypertensive patients. That study supported the relationship between nesfatin-1 and blood pressure. In contrast, another study with obese versus normal weight individuals did not find any relationship between nesfatin-1 and SBP or DBP. ${ }^{44}$ In the present study, no significant association was found between the nesfatin-1 concentrations and SBP or DBP.

Study limitations. The main limitation is the small sample size and recruitment of unhealthy controls (with fewer than 3 risk factors). This may explain the absence of a significant difference when comparing some variables between the 2 groups. Another limitation is that most of the patients were taking drugs for the treatment of different MetS risk factors, such as diabetes and hypertension, which would have influenced the results. This study was performed for partial fulfilment of master's degree and it was limited by time and the sample sizes justification was not done due to time limitation as a determining factor for number of samples.

In conclusion, our findings indicated a lower level of nesfatin-1 and a higher level of galanin in patients with MetS compared with control group. No significant relationship was found between nesfatin-1 and galanin. We recommend future studies with a larger sample size to clarify the exact role of these neuropeptides in the MetS process and address the link of galanin and nesfatin-1 with metabolic disturbances, such as obesity and insulin resistance.

Acknowledgment. Authors would like to thank King Abdulaziz City for Science and Technology (KACST), Riyadh, Saudi Arabia. This study was funded by KACST (Research No. 73-36). We would like to thank Scribendi (www.scribendi.com) for English language editing.

\section{References}

1. Kaur J. A comprehensive review on metabolic syndrome. Cardiol Res Pract 2014: 2014: 943162.

2. Ranasinghe P, Mathangasinghe Y, Jayawardena R, Hills A, Misra A. Prevalence and trends of metabolic syndrome among adults in the Asia-Pacific region: a systematic review. BMC Public Health 2017; 17: 101.
3. Bahijri S, Al Raddadi R. The importance of local criteria in the diagnosis of metabolic syndrome in Saudi Arabia. Ther Adv Endocrinol Metab 2013; 4: 51-59.

4. Sclafani A. Gut-brain nutrient signaling. Appetition vs. satiation. Appetite 2013; 71: 454-458.

5. Morton GJ, Meek TH, Schwartz MW. Neurobiology of food intake in health and disease. Nat Rev Neurosci 2014; 15: 367-378.

6. Ertuğrul ÖF. A novel type of activation function in artificial neural networks: trained activation function. Neural Networks 2018; 99: 148-157.

7. Mori M, Yamada M, Okada S, Hashimoto K, Satoh T, Shimizu $\mathrm{H}$ et al. Discovery of nesfatin-1 and overview of biological actions and new developments. Current Pharmaceutical Design 2013; 19: 6921-6928.

8. Elran-Barak R, Accurso E, Goldschmidt A, Sztainer M, Byrne C, Le Grange D. Eating patterns in youth with restricting and binge eating/purging type anorexia nervosa. International Journal of Eating Disorders 2014; 47: 878-883.

9. Dore R, Levata L, Lehnert H, Schulz C. Nesfatin-1: functions and physiology of a novel regulatory peptide. J Eat Disord 2017; 232: R45-R65.

10. Saldanha JF, Carrero JJ, Lobo JC, Stockler-pinto MB, Leal VO, Calixto A, et al. The newly identified anorexigenic adipokine nesfatin-1 in hemodialysis patients: are there associations with food intake, body composition and inflammation. Regul Pept 2012; 173: 82-85.

11. Khalili S, Khaniani S, Afkhami F, Derakhshan M. NUCB2/=nesfatin-1: a potent meal regulatory hormone and its role in diabetes. Egyptian Journal of Medical Human Genetics 2017; 18: 105-109.

12. Nakata M, Manaka K, Yamamoto S, Mori M, Yada T. Nesfatin-1 enhances glucose-induced insulin secretion by promoting $\mathrm{Ca}(2+)$ influx through L-type channels in mouse islet $\beta$-cells. Endocrine Journal 2011; 58: 305-313.

13. Ogiso K, Asakawa A, Amitani H, Nakahara T, Ushikai M, Haruta I, et al. Plasma nesfatin-1 concentrations in restrictingtype anorexia nervosa. Peptides 2011; 32: 150-153.

14. Ramanjaneya M, Chen J, Brown J, Tripathi G, Hallschmid M, Patel S, et al. Identification of nesfatin-1 in human and murine adipose tissue: a novel depot-specific adipokine with increased levels in obesity. Endocrinology 2010; 151: 3169-3180.

15. Fang P, Yu M, Shi M, Zhang Z, Sui Y, Guo L, et al. Galanin peptide family as a modulating target for contribution to metabolic syndrome. Gen Comp Endocrinol 2012; 179: 115-120.

16. Fang P, He B, Shi M, Kong G, Dong X, Zhu Y, et al. The regulative effect of galanin family members on link of energy metabolism and reproduction. Peptides 2015; 71: 240-249.

17. Śípková J, Kramáriková I, Hynie S, Klenerová V. The galanin and galanin receptor subtypes, its regulatory role in the biological and pathological functions. Physiol Res 2017; 66: 729-740.

18. Fang P, Shi M, Zhu Y, Bo P, Zhang Z. Type 2 diabetes mellitus as a disorder of galanin resistance. Exp Gerontol 2016; 73: 72-77.

19. Bu L, Yao Q, Liu Z, Tang W, Zou J, Qu S. Combined galanin with insulin improves insulin sensitivity of diabetic rat muscles. J Endocrinol 2014; 221; 157-165.

20. Fang P, Zhang L, Yu M, Sheng Z, Shi M, Zhu Y, et al. Activiated galanin receptor 2 attenuates insulin resistance in skeletal muscle of obese mice. Peptides 2018; 99: 92-98.

21. Nergiz S, Altınkaya S, Küçük M, Yüksel H, Sezer D, Kurt İ, et al. Circulating galanin and IL-6 concentrations in gestational diabetes mellitus. Gynecol Endocrinol 2014; 30: 236-240. 
22. Alberti K, Eckel R, Grundy S, Zimmer P, Cleeman J, Donato $\mathrm{K}$, et al. Harmonizing the metabolic syndrome: a joint interim statement of the International Diabetes Federation Task Force on Epidemiology and prevention; National Heart, Lung, and Blood Institute; American Heart Association; World Heart Federation; International Atherosclerosis Society; and International Association for the Study of Obesity. Circulation 2009; 120: 1640-1645.

23. Celi F, Bini V, Papi F, Santilli E, Ferretti A, Mencacci M, et al. Circulating acylated and total ghrelin and galanin in children with insulin-treated type 1 diabetes: relationship to insulin therapy, metabolic control and pubertal development. Clinical Endocrinology 2005; 63: 139-145.

24. Zhang Z, Fang P, Shi M, Gu C, Wang Y, Bo P, et al. Association between circulating levels of galanin and pre-pregnancy body mass index in patients with gestational diabetes mellitus. Eating Behaviors 2015; 19: 57-60.

25. Fang P, Yu M, Gu X, Shi M, Zhu Y, Zhang Z, et al. Low levels of plasma galanin in obese subjects with hypertension.J Endocrinol Invest 2017; 40: 63-68.

26. Fang P, Yu M, Guo L, Bo P, Zhang Z, Shi M. Galanin and its receptors: a novel strategy for appetite control and obesity therapy. Peptides 2012; 36: 331-339.

27. Boughton CK, Murphy KG. Can neuropeptides treat obesity? A review of neuropeptides and their potential role in the treatment of obesity. Br J Pharmacol 2013; 170: 1333-1348.

28. Fang P, Sun J, Wang X, Zhang Z, Bo P, Shi M. Galanin participates in the functional regulation of the diabetic heart. Life Sciences 2013; 92: 628-632.

29. Fang P, Yu M, Gu X, Shi M, Zhu Y, Zhang Z, et al. Circulating galanin and galanin like peptide concentrations are correlated with increased triglyceride concentration in obese patients. Clinica Chimica Acta 2016; 461: 126-129.

30. Algul S, Ozkan Y, Ozcelik O. Serum nesfatin-1 levels in patients with different glucose tolerance levels. Physiological Research 2016; 65: 979-985.

31. Su Y, Zhang J, Tang Y, Bi F, Liu Jn. The novel function of nesfatin-1: antihyperglycemia. Biochem Biophys Res Commun 2010; 391: 1039-1042.

32. Zhai T, Li S, Fan X, Tian Z, Lu X, Dong J. Circulating nesfatin-1 levels and type 2 diabetes: a systematic review and meta-analysis. J Diabetes Res 2017; 2017: 1-8.
33. Ademoglu En, Gorar S, Keskin M, Carlioglu A, Ucler R, Erdamar H, et al. Serum nesfatin-1 levels are decreased in pregnant women newly diagnosed with gestational diabetes. Arch Endocrinol Metab 2017; 61: 455-459.

34. Deniz R, Gurates B, Aydin S, Celik H, Sahin İ, Baykus Y, et al. Nesfatin-1 and other hormone alterations in polycystic ovary syndrome. Endocrine 2012; 42: 694-699.

35. Zhang Z, Li L, Yang M, Liu H, Boden G, Yang G. Increased plasma levels of nesfatin-1 in patients with newly diagnosed type 2 diabetes mellitus. Exp Clin Endocrinol Diabetes 2012; 120: 91-95.

36. LiZ, Gao L, Tang H, Yin Y, Xiang X, LiY, et al. Peripheral effects of nesfatin-1 on glucose homeostasis. PLoS One 2013; 8: e71513.

37. Anwar G, Yamamah G, Ibrahim A, El-Lebedy D, Farid T, Mahmoud R. Nesfatin-1 in childhood and adolescent obesity and its association with food intake, body composition and insulin resistance. Regulatory Peptides 2014; 188: 21-24.

38. Tan BK, Hallschmid M, Kern W, Lehnert H, Randeva HS. Decreased cerebrospinal fluid/plasma ratio of the novel satiety molecule, nesfatin-1/NUCB-2, in obese humans: evidence of nesfatin-1/NUCB-2 resistance and implications for obesity treatment. J Clin Endocrinol Metab 2011; 96: e669-e673.

39. Basar O, Akbal E, Koklu S, Kocak E, Tuna Y, Ekiz F, et al. A novel appetite peptide, nesfatin-1 in patients with non-alcoholic fatty liver disease. Scand J Clin Lab Invest 2012; 72: 479-483.

40. Sahin SB, Ayaz T, Cure MC, Sumer F, Ilkkilic K. Association of nesfatin-1 levels with fasting and postload glucoselevels in patients with hypothyroidism. J Exp Clin Med 2014; 31: 217-220.

41. Finelli C, Martelli G, Rossano R, Padula MC, La Sala N, Sommella L, Tarantino G. Nesfatin-1: role as possible new antiobesity treatment. EXCLIJ 2014; 13: 586-591.

42. Osaki A, Shimizu H. Peripheral administration of nesfatin-1 increases blood pressure in mice. Hypertension Research 2014; 37: 185-186.

43. Zhao Y, Ma X, Wang Q, Zhou Y, Zhang Y, Wu L, et al. Nesfatin-1 correlates with hypertension in overweight or obese Han Chinese population. Clin Exp Hypertens 2015; 37: 51-56.

44. Hamedani, N.K, Hosseinzadeh-Attar M.J, Hosseini M. The correlation between nesfatin-1 and blood pressure in healthy normal weight and obese adults. International Research Journal of Applied and Basic Sciences 2014; 8: 362-364. 